\title{
Describing the Profile of Patients on Concurrent Rifampin and Warfarin Therapy in Western Kenya: A Case Series
}

\author{
M. W. Maina S. D. Pastakia $\cdot$ I. Manji • \\ N. Kirui $\cdot$ C. Kirwa $\cdot$ Rakhi Karwa
}

Published online: 28 August 2013

(C) The Author(s) 2013. This article is published with open access at Springerlink.com

\begin{abstract}
Background Rifampicin's ability to induce hepatic enzymes is responsible for causing a clinically significant drug interaction with warfarin. Little data exists to guide clinicians on managing this interaction, especially in SubSaharan Africa where many patients are exposed to this combination due to a higher burden of tuberculosis.

Objective The objective of the case series is to provide insight to practicing clinicians of the unique dynamics of this drug interaction in resource-constrained settings. The case series will provide details on commonly encountered scenarios and the dosage adjustments required to maintain a therapeutic INR.

Methods A retrospective chart review was conducted of patients attending the Moi Teaching and Referral Hospital
\end{abstract}

M. W. Maina - S. D. Pastakia - I. Manji · N. Kirui · R. Karwa Moi Teaching and Referral Hospital-Anticoagulation Clinic, P.O Box 3-30100, Eldoret, Kenya

M. W. Maina · S. D. Pastakia · I. Manji - R. Karwa Department of Pharmacy Practice, Purdue University College of Pharmacy, West Lafayette, IN, USA

S. D. Pastakia · R. Karwa

Department of Pharmacology, Moi University School of Medicine, Eldoret, Kenya

S. D. Pastakia · R. Karwa

Indiana University School of Medicine, Indianapolis, USA

I. Manji · C. Kirwa

Academic Model Providing Access to Healthcare (AMPATH), Eldoret, Kenya

R. Karwa $(\square)$

Wishard Health Services, W7555 Myers Building, 1001 W. 10th Street, Indianapolis, IN 46202-2879, USA

e-mail: rakhikarwa@gmail.com anticoagulation clinic in Eldoret, Kenya. Patients were included if they had a history of concurrent rifampicin and warfarin therapy and a minimum follow up of 2 months. Descriptive statistics were used to explain the demographic characteristics, time to therapeutic INR and average weekly warfarin dose. The inference on proportions test was conducted to compare the time in the therapeutic range (TTR) for patients on concurrent rifampicin to the rest of the patients not receiving rifampicin in the clinic.

Results Of the 350 patient charts evaluated, 10 met the inclusion criteria. The median percentage increase of the weekly warfarin dose from baseline was $15.7 \%$. For the patients in this analysis, the median TTR was $47 \%$.

Discussion Patients on concurrent therapy should be rigorously monitored with regular INR checks and warfarin dosage adjustments. Empiric dosage adjustments of warfarin should be avoided but patient characteristics can aid in understanding the alterations seen in INR.

\section{Background}

Vitamin K antagonists (VKAs), such as warfarin, form the foundation of anticoagulation therapy due to their proven effectiveness and affordability [1]. For decades, VKAs have been indicated for both the prophylaxis and treatment of venous thromboembolism (VTE) and thromboembolic complications associated with atrial fibrillation and cardiac valve replacement. In addition, they have been shown to reduce the risk of death, recurrent myocardial infarction and thromboembolic events such as stroke [2]. Despite their benefits and widespread use, many challenges are faced when using warfarin. These include variable interpatient warfarin dose response due to age, co-morbidities, liver function, albumin level, genetic polymorphism in 
enzymes, and numerous drug-drug/drug-diet interactions [1, 3-5]. Consequently, close monitoring using the international normalized ratio (INR) and patient specific dosing must be applied when utilizing warfarin [5].

Because of its pharmacokinetic and metabolic profile, warfarin is prone to having drug-drug interactions affecting the intensity of monitoring and clinical efficacy. Warfarin is a racemic mixture of both $R$ and $S$ enantiomers. The enantiomers differ in that R-warfarin is less potent and has a longer half-life when compared to S-warfarin. In addition, R-warfarin is metabolized by the enzymes cytochrome P450 (CYP) 1A2 and CYP 3A4, whereas $\mathrm{S}$-warfarin is metabolized by CYP 2C9 [6]. It is noted that rifampicin is a potent and nonspecific inducer of the hepatic CYP450 oxidative enzyme system. Although it is recognized that rifampicin causes marked enzyme induction of CYP 3A4, it is still considered to have an enhanced effect on the metabolism of both enantiomers [7]. Importantly, the accelerated clearance can lead to compromised efficacy and reduced anticoagulant effects of warfarin [8]. The clinically significant alterations in the INR can create the need for more intense monitoring and large warfarin dose adjustments.

Currently, only seven case reports have been published describing the interaction between warfarin and rifampicin, all of which come from the developed world where tuberculosis (TB) rates are much lower [5, 9-14]. Due to its efficacy and relative affordability, rifampicin is part of the first line regimen for treatment of TB [15]. With an increased prevalence of TB in developing countries, it is likely that there is increased use of rifampicin, and thus, more concern for the potential drug-drug interactions with warfarin in these settings.

According to a study carried out on the global burden of $\mathrm{TB}, 10$ of the 22 countries with the highest incidence rates per capita of TB are in Africa. In the same report, Kenya is ranked 15 th in the list of 22 high-burden TB countries, with an incidence of 288 per 100,000 population [16]. The Kenya National Leprosy and TB Treatment Guidelines (2009) recommend the use of rifampicin, isoniazid, ethambutol and pyrazinamide as first line therapy for 2 months, followed by 4 months of rifampicin and isoniazid. In Kenya, all TB medications in the standard medication regimen are provided for free by the ministry of health in the form of fixed dose combinations. With the reliance on a 6-month $\mathrm{TB}$ regimen, all patients are to receive TB medications via directly observed therapy, which typically occurs in the presence of a relative or friend that lives in close proximity to the TB infected patient [17].

Because of the higher prevalence of TB and emerging availability of anticoagulation services in this setting, there exists a growing population of patients who are facing this drug interaction [18, 19]. Even though anticoagulation clinics have been shown to improve patient outcomes when compared to individual physician care, the limited data concerning this drug-drug interaction in this population presents an enormous challenge to clinicians providing care to patients on concomitant rifampicin and warfarin therapy [2]. Without data from patients receiving care in developing countries, clinicians have to rely primarily on the previously published case reports conducted only in developed countries, some of which suggest the need to increase warfarin doses by greater than 100-200\% [5, 9, 10].

The objective of this case series is to provide insight to practicing clinicians on the unique dynamics of the drug interaction between rifampicin and warfarin therapy in a resource-constrained setting in western Kenya. The case series will provide details on commonly encountered scenarios in these settings and the adjustments made to maintain a therapeutic INR. With the high numbers of TB infected patients within this setting, this represents one of the largest case series on this often encountered drug interaction and the first which considers the unique characteristics of patients within a rural resource-constrained setting.

\section{Methods}

The study is a retrospective chart review of patients receiving concurrent anti-TB medications containing rifampicin and oral anticoagulation therapy with warfarin.

This study was conducted in a pharmacist-managed anticoagulation clinic within the Moi Teaching and Referral Hospital (MTRH) in Eldoret, Kenya. The anticoagulation clinic was established through a partnership formed by the Purdue University College of Pharmacy, the Academic Model Providing Access to Healthcare (AMPATH), MTRH and Moi University School of Medicine [20]. The clinic was developed as AMPATH expanded its scope of practice from the human immunodeficiency virus (HIV) pandemic to chronic disease management and primary health care. Since the clinic's inception in December 2008, it has served over 700 patients and currently has more than 350 active patients.

The majority of patients are enrolled into the anticoagulation clinic through referrals from MTRH clinicians providing health services in the public inpatient and outpatient clinics. Most patients are referred from the cardiology, obstetrics/gynecology, internal medicine and hematology/oncology departments. The most common indications for anticoagulation in the clinic include VTE, valvular damage secondary to rheumatic heart disease (RHD) and atrial fibrillation. Patients with mechanical 
heart valves and other cardiomyopathies also receive anticoagulation therapy within the clinic [18].

Upon enrollment into the anticoagulation clinic, an initial encounter form is completed detailing the patient's demographic data, indications for warfarin and intended duration of therapy, co-morbidities, and concurrent medications. The patients routinely visit the clinic for assessment, which includes point of care INR testing, assessment of dietary vitamin $\mathrm{K}$ intake, pill count based assessment for adherence, refill of warfarin into pill boxes and monitoring of adverse events due to warfarin such as bleeding. Warfarin doses are adjusted based on these factors using a comprehensive protocol based on the American College of Chest Physician Guidelines (2008) [21]. Information on the patient encounter is recorded on a standardized form, which is completed at every visit. The frequency of patient visits is dependent upon the consistency of their INR within the therapeutic range and accessibility to the clinic [18].

The study included all patients on concurrent warfarin and rifampicin therapy enrolled in the clinic from May 2009 to June 2011 and on follow-up at the anticoagulation clinic for a minimum of 2 months. Patients on antiretroviral therapy were excluded due to the potential for additional drug interactions, which would limit the ability to focus on the impact of rifampicin. Data was collected from the patient charts that contained their initial encounter form and routine assessment forms. Patients were assessed for time to therapeutic INR, average weekly warfarin dose on attaining therapeutic INR, time in therapeutic range (TTR) and level of adherence. Institutional Review Board (IRB) approval was obtained from the local institutional review and ethics committee at MTRH/Moi University and the Indiana University-Purdue University Indianapolis (IUPUI) IRB.

In this study, time to therapeutic INR is defined as the time taken to achieve two consecutive therapeutic INRs. The average weekly warfarin doses on attaining therapeutic INR were calculated with similar considerations. Time in therapeutic range (TTR) is calculated using the linear interpolation method described by Rosendaal et al. [22] and weighted by the duration of follow-up of each patient. The model assumes that the INR changes linearly between measurements and estimates the percentage of time spent in the therapeutic range. Adherence to therapy is generally defined as the extent to which patients take medications as prescribed by their health care providers. It may also include details on the patient's dose taking tendencies [23]. In this case series, our definition encompasses both and therefore refers to adherence with the prescribed warfarin regimen as indicated by the healthcare provider. In order to improve outcomes from the, often complicated, warfarin dosing regimens, all of the warfarin is dispensed in pill boxes with adherence assessed via pill box based pill counts at each clinic visit. The adherence of all other medications was based on patient self-report of adherence and utilization of concomitant medications. Descriptive statistics were utilized to describe the demographic characteristics of the population in addition to the anticoagulation clinic specific metrics. The inference on proportions test was utilized to compare the TTR between the group concurrently treated with rifampicin and the rest of the anticoagulation clinic [19]. Stata $11.0^{\circledR}$ was used to perform all statistical analyses.

\section{Results}

From the 350 charts reviewed, 10 met the inclusion criteria as seen in the flow chart of enrollment in Fig. 1. As described in the summary of patient characteristics in Table 1 , the majority of the patients included within this analysis were female $(60 \%)$ with the main indication for anticoagulation being VTE ( $80 \%)$. The median percentage increase of the weekly warfarin dose was $15.7 \%$ with a median weekly dose of $73.1 \mathrm{mg}$. For the patients in this analysis, the median TTR was $47 \%$ (95\% CI 12-74). Prior analyses of the performance of the rest of the anticoagulation clinic revealed an average TTR of $62 \%(95 \%$ CI 54-69). The inference on proportions test did not illustrate a statistically significant difference between the TTR of the rest of the anticoagulation clinic and TTR of the group of patients on rifampicin; however, this is largely

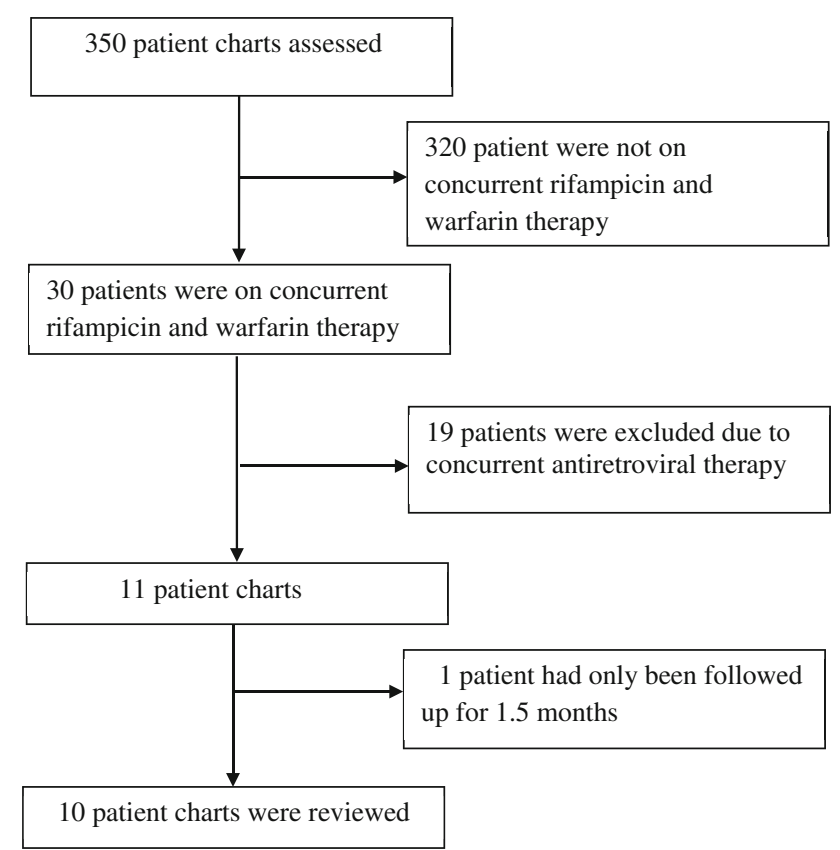

Fig. 1 Flowchart of the study 


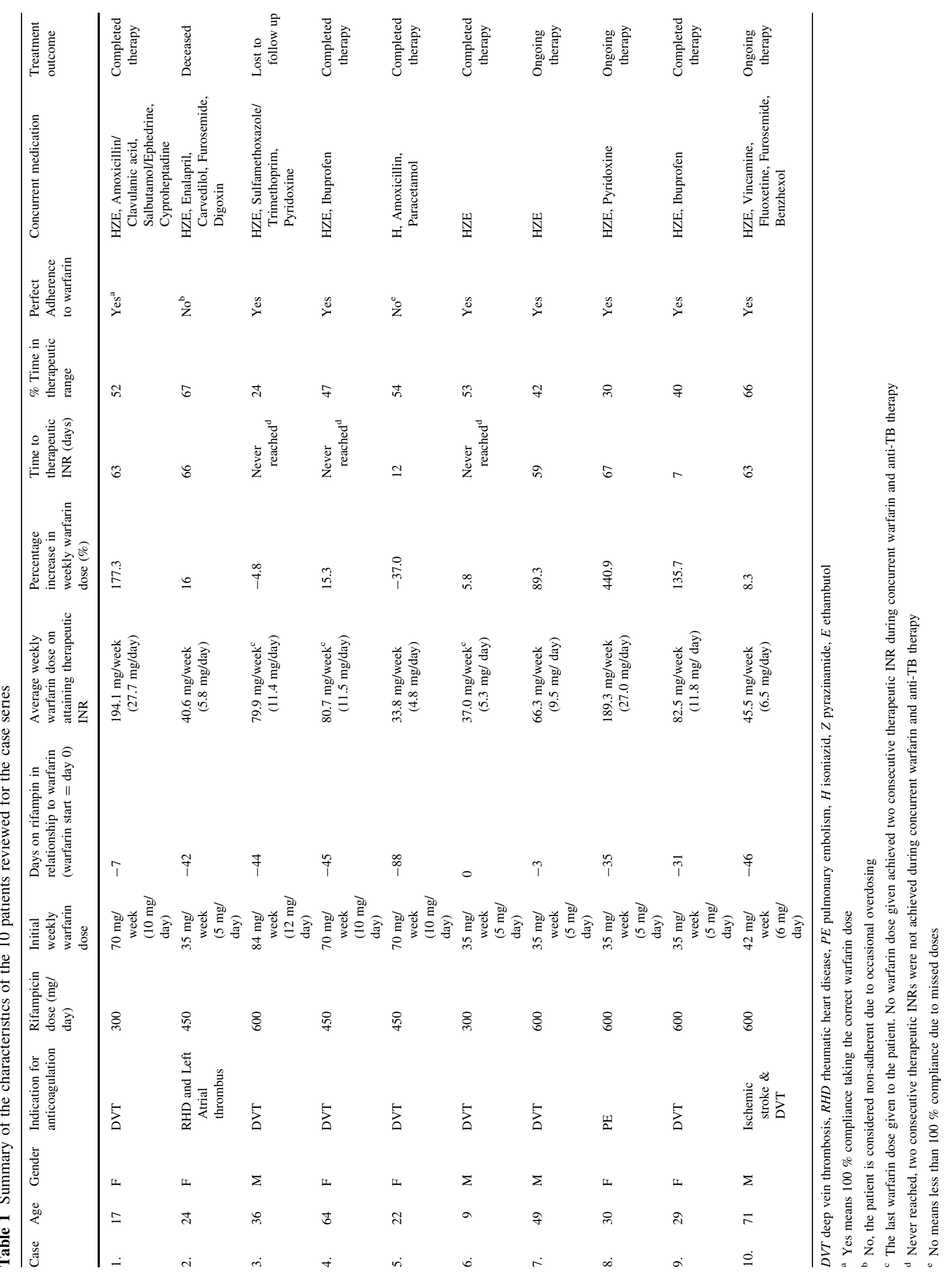


Table 2 Measures of central tendency for variables from the cases

\begin{tabular}{|c|c|}
\hline Variable & $\begin{array}{l}\text { Median, } \\
\text { interquartile } \\
\text { range }\end{array}$ \\
\hline Age (years) & $29.5(20.75-52.75)$ \\
\hline $\begin{array}{l}\text { Percentage increase in weekly warfarin dose to } \\
\text { achieve therapeutic INR (\%) }\end{array}$ & $15.7(3.15-146.1)$ \\
\hline $\begin{array}{l}\text { Median weekly warfarin dose on attaining a } \\
\text { therapeutic INR }\end{array}$ & $73.1(38.8-81.6)$ \\
\hline $\begin{array}{l}\text { Median daily warfarin dose on attaining a } \\
\text { therapeutic INR }\end{array}$ & $10.4(5.5-11.7)$ \\
\hline Days to therapeutic INR (days) & $61^{\mathrm{a}}(18-65.25)^{\mathrm{a}}$ \\
\hline Time in therapeutic range (TTR) (\%) & $47(30-54)$ \\
\hline
\end{tabular}

${ }^{a}$ Includes only the patients who reached therapeutic INR during their anticoagulation therapy $(n=8)$

due to the difference in sample size between the two comparison groups (17\% difference between groups, $95 \%$ CI $[-15-48], P=0.23)$. Table 2 shows the central tendencies for the anticoagulation clinic specific variables from the cases. The majority of the patients were initiated on $35 \mathrm{mg} /$ week of warfarin with the exception of cases 1, 4 and 5 who were initiated on $70 \mathrm{mg} /$ week. The differences in the initial weekly warfarin dose were based on variable practices of the primary physicians managing those cases, as certain providers prefer starting at higher doses prior to the patient enrollment in the clinic.

Further assessment of the findings reveal certain clinically relevant trends including, but not limited to, the influence of age, timing of rifampicin and warfarin use, impact of comorbid conditions, and effect of concomitant medication use. When looking at patients at the extremes of age (case 6 [9 years old] and case 10 [71 years old]), a smaller percentage increase in the weekly warfarin dose is seen. The dramatic impact of the timing of rifampicin use and warfarin therapy can be seen when looking at the results of case 1 and case 7 as these cases required a relatively large increase in their weekly warfarin dose of 177.3 and $89.3 \%$ respectively. In both of these cases, warfarin therapy was started within 2 weeks of starting rifampicin. The impact of comorbidities on warfarin dosing in the presence of rifampicin can be seen amongst cases 2 (RHD), 3 (HIV [not on antiretroviral therapy]), 4 (severe osteoarthritis), and 10 (cerebral infarct). These patients required only modest increases or decreases from their original dosing regimen.

\section{Discussion}

This case series highlights the highly variable response to the drug interaction between rifampicin and warfarin amongst rural resource-constrained patients in western
Kenya. While much of this variability can be partially explained by the comorbid conditions and other anticoagulation modifying characteristics of patients, this case series highlights the extreme unpredictability of this interaction and need for individualized therapy. Patients tended to require a higher than normal weekly dose (73.1 mg per week (10.4 mg/day). However, the interquartile range for these findings was quite large, limiting the ability to provide uniform dosing guidance for future patients that may encounter this drug interaction. The TTR for patients receiving rifampicin and warfarin was lower than the TTR for patients not utilizing rifampicin in clinic. Although, the difference in TTR was not statistically significant, it highlights the added difficulty in managing anticoagulation therapy in these patients. In addition, distinct patient characteristics such as, age, start dates of rifampicin in relation to warfarin, and co-morbid conditions likely play a role in the intricacy of dosing and monitoring requirements of these patients.

The findings regarding the impact of age on warfarin dosing are supported by the well-documented physiological changes that occur in these age groups. In pediatrics, the hemostatic system is a dynamic and evolving entity with both quantitative and qualitative changes in its components. The changes affect the concentration and functionality of the blood clotting factors. The differences in the system are marked in neonates and infants and continue to mature during childhood until reaching full development during adolescence [24, 25]. These changes affect the response to anticoagulant agents. Also, in studies carried out in children, age has been shown to affect the pharmacokinetic and pharmacodynamic responses to anticoagulants [26, 27]. This may possibly explain the small change in weekly warfarin dose in case 6 . On the other extreme, the geriatric population (age $>65$ years; Case 10) is associated with lower than usual warfarin dose requirements, which may be attributed to impaired enzyme induction in the elderly [2, 28]. Clinicians should be cautious when adjusting warfarin doses in patients at the extremes of age due to the variation in the hemostatic system and drug pharmacokinetics.

In addition to the age of the patient, the start date of rifampicin in relationship to warfarin utilization can have a direct impact on the degree of necessary dosing adjustments of the anticoagulant. In patients who started rifampicin therapy within two weeks of starting warfarin, the impact of rifampicin timing was quite pronounced as most patients required large increases in their warfarin dose to compensate for the emerging induction of warfarin metabolism. The increase in weekly warfarin dose may be attributable to the time required to induce hepatic enzymes involved in warfarin's metabolism by rifampicin, typically seen 1 to 2 weeks after starting long-term rifampicin 
therapy $[10,29]$. These cases can be contrasted with cases 2, 3 and 4 whose warfarin therapy was started more than 2 weeks after the initiation of rifampicin. The percentage increase in weekly warfarin dose in these patients was not as dramatic $(16.0 \%,-4.8 \%$ and $15.3 \%$ respectively). However, exceptions to this observation exist such as that seen in case 8 . Case 8 , a 38 year-old female on warfarin therapy due to pulmonary embolism and DVT, was on rifampicin treatment for more than two weeks before warfarin was started, and yet showed a $440.9 \%$ increase in weekly warfarin dose from the initial starting dose. Compared to cases 2, 3 and 4, described above, the timing of warfarin initiation in relation to the commencement of rifampicin therapy in case 8 should have resulted in a less dramatic percent increase in the warfarin dose. Clinicians should therefore anticipate a large percentage increase in weekly warfarin dose and should frequently assess patients whose warfarin therapy is started simultaneously or within 2 weeks of initiating rifampicin. Empiric dose adjustments based on the start date of rifampicin are not recommended.

Table 1 also highlights the potential impact of other concomitant interacting medications as several of the patients were on antibiotics (amoxicillin/clavulanic acid, sulfamethoxazole/trimethoprim), cardiovascular medications (furosemide), pain medications (paracetamol, ibuprofen) and mental health medications known to alter the response to warfarin [30-36]. Without an appropriate control group, it is difficult to determine how these medications might have impacted the response to the drug interaction between warfarin and rifampicin. In addition, many of these patients had other co-morbid conditions, which can increase the complexity of warfarin therapy. Such patients are also more likely to have unpredictable variations in their overall health status and concurrent medications that may potentially interact with warfarin, requiring more intense monitoring of INR and adverse drug reactions [37].

This study possesses certain key limitations largely related to its retrospective nature and reliance on data obtained during the routine clinical encounter. While the study was able to definitively determine the adherence to warfarin, adherence to other medications was based purely on patient self-report. With the case series design of this investigation, the ability to form conclusive recommendations on the dosing of rifampicin in different populations is difficult as a comparison control group is lacking and the patient population is small.

\section{Conclusion}

With access to healthcare infrastructure in sub-Saharan Africa continuing to grow, there is an emerging need for contextualized research describing the unique dynamics and responses to therapy in these populations. As shown within this case series, previous case report findings and recommendations from resource-rich settings, which illustrated the need to drastically increase the warfarin dose, would result in significant overdoses for the majority of patients within this setting. Based on the findings, it is recommended that patients on concomitant warfarin and rifampicin therapy be rigorously monitored with regular INR checks and warfarin dose adjustments. Empiric dosage changes should be discouraged due to the unpredictability of response to this exigent interaction. Also, more studies should be carried out to enhance the comprehension of factors influencing the variation in warfarin dose in such patients in the sub-Saharan African population.

Acknowlegments This research was supported in part by a grant to the USAID-AMPATH Partnership from the United States Agency for International Development as part of the President's Emergency Plan for AIDS Relief (PEPFAR) in addition to support from the Indiana Hemophilia and Thrombosis Center (Indianapolis, Indiana, USA).

Funding and Conflict of interests This research was supported in part by a grant to the USAID-AMPATH Partnership in addition to support from the Indiana Hemophilia and Thrombosis Center (Indianapolis, Indiana, USA)

All authors declare they have no conflicts of interest.

Open Access This article is distributed under the terms of the Creative Commons Attribution Noncommercial License which permits any noncommercial use, distribution, and reproduction in any medium, provided the original author(s) and the source are credited.

\section{References}

1. Lowery S, Haley K, Bussey HI. Oral anticoagulation: challenges in the case-management setting. Lippincott's Case Manag. 2005;10(1):39-50.

2. Ageno W, Gallus AS, Wittkowsky A, Crowther M, Hylek EM, Palareti G; American College of Chest Physicians. Oral anticoagulant therapy: Antithrombotic Therapy and Prevention of Thrombosis, 9th ed. American College of Chest Physicians Evidence-Based Clinical Practice Guidelines. Chest. 2012;141(2 Suppl):e44S-88S.

3. Wells PS, Holbrook AM, Crowther NR, et al. Interactions of warfarin with drugs and food. Ann Intern Med. 1994;121:676-83.

4. Harder S, Thürmann P. Clinically important drug interactions with anticoagulants. An update. Clin Pharmacokinet. 1996;30:416-44.

5. Krajewski KC. Inability to achieve a therapeutic INR value while on concurrent warfarin and rifampin. J Clin Pharmacol. 2010; 50:710-3.

6. Cropp JS, Bussey HI. A review of enzyme induction of warfarin metabolism with recommendations for patient management. Pharmacotherapy. 1997;17(5):917-28.

7. Niemi M, Backman JT, Fromm MF, et al. Pharmacokinetic interactions with rifampicin: clinical relevance. Clin Pharmacokinet. 2003;42:819-50.

8. O'Reilly RA. Interaction of chronic daily warfarin therapy and rifampin. Ann Intern Med. 1975;83:506-8. 
9. Kim KY, Epplen K, Foruhari F, Alexandropoulos H. Update on the interaction of rifampin and warfarin. Prog Cardiovasc Nurs. 2007 Spring;22(2):97-100.

10. Lee CR, Thrasher KA. Difficulties in anticoagulation management during coadministration of warfarin and rifampin. Pharmacotherapy. 2001;21(10):1240-6.

11. Casner PR. Inability to attain oral anticoagulation: warfarinrifampin interaction revisited. South Med J. 1996;89(12):1200-3.

12. Almog S, Martinowitz U, Halkin H, Bank HZ, Farfel Z. Complex interaction of rifampin and warfarin. South Med J. 1988;81 (10): 1304-6.

13. Self TH, Mann RB. Interaction of rifampin and warfarin. Chest. 1975;67(4):490-1.

14. Romankiewicz JA, Ehrman M. Rifampin and warfarin: a drug interaction. Ann Intern Med. 1975;82(2):224-5.

15. World Health Organization. Treatment of tuberculosis guidelines. Fourth Edition. 2010. http://whqlibdoc.who.int/publications/ 2010/9789241547833_eng.pdf. Accessed 22 July 2013.

16. World Health Organization. Global Tuberculosis Report 2012. http://apps.who.int/iris/bitstream/10665/75938/1/ 9789241564502_eng.pdf. Accessed 22 July 2013.

17. Division of Leprosy, Tuberculosis and Lung Disease. DLTLD Guidelines on management of leprosy and tuberculosis. March 2009. http://www.nltp.co.ke/docs/DLTLD_Treatment_Guide lines.pdf. Accessed 22 July 2013.

18. Pastakia SD, Crisp WI, Schellhase EM, et al. Implementation of a pharmacist managed anticoagulation clinic in Eldoret, Kenya. South Med Rev. 2010;3:20-3.

19. Manji I, Pastakia SD, DO AN, et al. Performance outcomes of a pharmacist-managed anticoagulation clinic in the rural, resourceconstrained setting of Eldoret, Kenya. J Thromb Haemost. 2011;9:2215-20.

20. Pastakia SD, Schellhase EM, Jakait B. Collaborative partnership for clinical pharmacy services in Kenya. Am J Health Syst Pharm. 2009;66:1386-90.

21. Ansell J, Hirsh J, Hylek E, et al. American College of Chest Physicians. Pharmacology and management of the vitamin K antagonists: American College of Chest Physicians EvidenceBased Clinical Practice Guidelines (8th Edition). Chest. 2008; 133:160S-98S.

22. Rosendaal FR, Cannegieter SC, van der Meer FJ, et al. A method to determine the optimal intensity of oral anticoagulant therapy. Thromb Haemost. 1993;69:236-9.
23. Osterberg L, Blaschke T. Adherence to medication. N Engl J Med. 2005;353:487-97.

24. Monagle P, Barnes C, Ignjatovic V, et al. Developmental haemostasis. Impact for clinical haemostasis laboratories. Thromb Haemost. 2006;95:362-72.

25. Payne JH. Aspects of anticoagulation in children. Br J Haematol. 2010;150:259-77.

26. Streif W, Andrew M, Marzinotto V, et al. Analysis of warfarin therapy in pediatric patients: A prospective cohort study of 319 patients. Blood. 1999;94:3007-14.

27. Kuhle S, Massicotte $P$, Dinyari $M$, et al. Dose-finding and pharmacokinetics of therapeutic doses of tinzaparin in pediatric patients with thromboembolic events. Thromb Haemost. 2005; 94:1164-71.

28. Twum-Barima Y, Finnigan T, Habash AI, et al. Impaired enzyme induction by rifampicin in the elderly. Br J Clin Pharmacol. 1984;17:595-7.

29. Michalets EL. Update: clinically significant cytochrome P-450 drug interactions. Pharmacotherapy. 1998;18:84-112.

30. Woolfrey S, Gammack NS, Dewar MS, et al. Fluoxetine-warfarin interaction. BMJ. 1993;307:241.

31. Glasheen JJ, Fugit RV, Prochazka AV. The risk of overanticoagulation with antibiotic use in outpatients on stable warfarin regimens. J Gen Intern Med. 2005;20:653-6.

32. Laizure SC, Madlock L, Cyr M, et al. Decreased hypoprothrombinemic effect of warfarin associated with furosemide. Ther Drug Monit. 1997;19:361-3.

33. Davies RO, Gomez HJ, Irvin JD, et al. An overview of the clinical pharmacology of enalapril. Br J Clin Pharmacol. 1984;18 (Suppl 2):215S-29S.

34. Bristow MR. Pathophysiologic and pharmacologic rationales for clinical management of chronic heart failure with beta-blocking agents. Am J Cardiol. 1993;71:12C-22C.

35. van Dijk KN, Plat AW, van Dijk AA, et al. Potential interaction between acenocoumarol and diclofenac, naproxen and ibuprofen and role of CYP2C9 genotype. Thromb Haemost. 2004;1:95-101.

36. Hughes GJ, Patel PN, Saxena N. Effect of acetaminophen on international normalized ratio in patients receiving warfarin therapy. Pharmacotherapy. 2011;31:591-7.

37. Torn M, Bollen WL, van der Meer FJ, et al. Risks of oral anticoagulant therapy with increasing age. Arch Intern Med. 2005; 165:1527-32. 